\title{
Comparação entre os diagnósticos pela inspeção sanitária post-mortem e histopatologia da pneumonia enzoótica suína: estudo de caso-controle
}

\author{
Comparison between diagnosis by sanitary inspection and \\ histophatology of swine enzootic pneumonia: case-control study
}

\author{
Kênia de Fátima Carrijo, ${ }^{*}$ Elmiro Rosendo Nascimento, ${ }^{* *}$ lacir Francisco dos Santos, ${ }^{* * *}$ Elias Chagas, ${ }^{* * *}$ \\ Lucas Kuiava, ${ }^{* * * *}$ Rogerio Tortelly ${ }^{* * * * * *}$
}

\begin{abstract}
Resumo
Em estudo epidemiológico tipo caso-controle realizado em matadouro-frigorífico do Oeste de Santa Catarina, observou-se a ocorrência de pneumonia enzoótica em suínos, diagnosticada sob inspeção sanitária e histopatologia, bem como se o peso da carcaça quente modificaria em função da enfermidade. O grupo caso foi representado por 69 suínos portadores da enfermidade e o grupo controle, por 69 animais sem a enfermidade, identificados por técnicos do Serviço de Inspeção Federal (SIF) com base em características morfológicas previamente padronizadas. Dos animais positivos para a enfermidade na inspeção sanitária, $75,36 \%$ deles (52/69) tiveram diagnóstico positivo na microscopia. No grupo controle (negativos na inspeção sanitária), 14,49\% (10/69) também foram positivos na microscopia, não havendo diferença significante entre os dois tipos de diagnóstico $(P>0,05)$, os quais concordaram em $62 \%$ pelo método de Kappa. A média de peso da carcaça quente no grupo controle foi $2,35 \mathrm{~kg}$ maior que no grupo caso, porém essa diferença de peso não foi significativa $(P>0,05)$.
\end{abstract}

Palavras-chave: pneumonia enzoótica suína, inspeção sanitária, histopatologia, suínos.

\begin{abstract}
In an epidemiological case-control study carried out in the west of Santa Catarina state slaughterhouses, the occurrence of Enzootic Pneumonia in swine slaughtered under sanitary inspection as well as the effect of this disease on hot carcass weight was accused. The lungs examined were identified by technicians of the Federal Inspection Service based on the presence of standardized morphological characteristics. Using the case-control design, 69 swine identified as having Enzootic Pneumonia were compared with 69 swine without Enzootic Pneumonia. Within the animals with Enzootic Pneumonia diagnosed by Sanitary Inspection, 75.36\% (52/69) were diagnosed as positive by histopathology. Within the animals without Enzootic Pneumonia diagnosed by Sanitary Inspection, $14.49 \%(10 / 69)$ were diagnosed as positive by histopathology $(P>0.05)$ without significant differences between them, but with Kappa agreement of $62 \%$. The average of hot carcass weight within control group was 2.35 $\mathrm{Kg}$ bigger than case group, but this difference was not significative $(P>0,05)$.
\end{abstract}

Keywords: Swine Enzootic Pneumonia, sanitary inspection, histopathology, swine.

\section{Introdução}

A pneumonia enzoótica suína (PES) causada pelo agente Mycoplasma hyopneumoniae representa a causa mais comum de perdas associadas às doenças respiratórias na produção intensiva de suínos (Zimmermann e Plonait, 1997).
Trata-se de uma enfermidade muito contagiosa, que se manifesta clinicamente por tosse seca não produtiva, alta morbidade, baixa mortalidade e principalmente, atraso no ganho de peso. Além disso, pode desencadear complicações broncopulmonares purulentas em função do aumento da susceptibilidade para outros agentes que causam pneumonia

* Programa de Pós-Graduação em Medicina Veterinária - Higiene Veterinária e Processamento Tecnológico de Produtos de Origem Animal. Autor para correspondência: Kênia de Fátima Carrijo. Endereço: Av. Alm. Ary Parreiras, 467, apto. 203, Jardim Icaraí, Niterói, RJ. CEP: 24.230322. E-mail: keniafcarrijo@yahoo.com.br.

** Departamento de Saúde Coletiva Veterinária e Saúde Pública da Faculdade de Veterinária - Universidade Federal Fluminense. Rua Vital Brazil Filho, 64- Vital Brazil, Niterói RJ, Brasil. CEP: 24230-340.

*** Departamento de Tecnologia dos Alimentos da Faculdade de Veterinária - Universidade Federal Fluminense.

**** Fiscal Federal Agropecuário - MAPA.

***** Faculdade de Medicina Veterinária - Universidade de Passo Fundo.

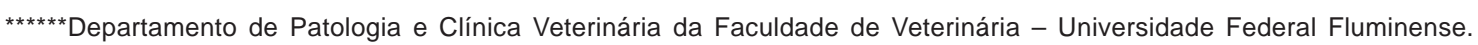


em função do quadro de imunodepressão que se instala (Sobestiansky et al., 1999).

Segundo Sobestiansky et al. (2001), apenas 5\% dos suínos de terminação apresentam sinais evidentes de pneumonia, geralmente quando complicadas por agentes secundários, visto que no caso de uma infecção somente pelo $M$. hyopneumoniae, a manifestação clínica pode passar despercebida. Com isso, o produtor imagina que são somente esses os animais doentes. Entretanto, nos demais, aparentemente saudáveis, a doença pode estar cursando na sua forma subclínica e em níveis economicamente críticos, ocasionando queda no rendimento e condenação de carcaças na indústria.

Por ser uma doença de ampla distribuição e de alta prevalência, o médico-veterinário tem a obrigação de conhecer perfeitamente a situação dos plantéis aos quais presta assistência, para estabelecer programas eficientes de controle. Antes de recomendar qualquer medida, é importante conhecer como a doença se comporta no rebanho, através de exames laboratoriais como sorologia e reação de polimerase em cadeia e acompanhamento através das alterações anátomo-patológicas dos lotes de suínos no matadouro (Sobestiansky et al., 1999). É no exame postmortem, realizado nos frigoríficos por fiscais sanitários, que as lesões por PES podem ser diagnosticadas mediante exames macroscópicos, indicando a situação de saúde do rebanho e gerando dados que podem ser de grande importância em estudos epidemiológicos da enfermidade (Maes et al., 2001; Walker e Bilkei, 2006; Martínez et al, 2007). Piffer (1997) ainda enfatiza que o acompanhamento das alterações anátomo-patológicas em frigoríficos é uma das mais importantes fontes de informações conhecidas para a avaliação sanitária dos suínos, com a finalidade de identificar e quantificar a prevalência das doenças, bem como a severidade das lesões.

Dessa maneira, não há dúvidas de que os dados obtidos pela Inspeção Sanitária no matadouro constituem um importante subsídio para a avaliação das perdas econômicas, bem como contribuem para o estabelecimento de um método de diagnóstico mais adequado.

Do ponto de vista econômico, esta enfermidade causa prejuízos muito elevados. Armstrong (1994) estimou que, nos Estados Unidos, as perdas anuais podem estar por volta de 200 a 330 milhões de dólares em função da redução no índice de crescimento quando o animal se infecta ainda jovem, e piora da conversão alimentar, que afeta diretamente o ganho médio de peso diário e a qualidade das carcaças.

$\mathrm{Na}$ literatura, apesar de existirem numerosos relatos comparando resultados de diagnóstico das lesões pulmonares na inspeção sanitária com resultados sorológicos (Mousing et al., 1990; Bahnson et al., 1994; Morris et al., 1995), são escassos os trabalhos que comparam a eficiência do diagnóstico macroscópico com a histopatologia (microscópico).

A partir dos aspectos mencionados, o presente trabalho tem como objetivo comparar o grau de acerto e concordância dos diagnósticos de pulmões com lesões sugestivas de PES na Inspeção Sanitária post-mortem com a histopatologia, mediante a realização de estudo observacional do tipo caso- controle e verificar se o peso da carcaça quente se alteraria em função da enfermidade.

\section{Material e método}

Foram utilizadas amostras de pulmões de suínos abatidos em matadouro-frigorífico sob Inspeção Federal, do Oeste de Santa Catarina, durante o mês de março de 2007. O estabelecimento abate em média 1800 suínos por dia, procedentes de vários municípios da microrregião Oeste de Santa Catarina, vacinados contra a pneumonia enzoótica. Foram coletados em comum acordo com o Fiscal Sanitário, 69 amostras de pulmões com lesões macroscópicas características de PES e 69 amostras de pulmões que não apresentavam tais lesões. Para cada amostra caso, era obtida em seguida uma amostra controle de forma alternada, com o cuidado de que fossem do mesmo plantel. As amostras utilizadas no grupo caso foram retiradas do lobo pulmonar que apresentava maior extensão de lesão macroscópica. No grupo controle, foram coletados no lobo correspondente ao coletado no grupo caso. Em seguida as amostras foram acondicionadas individualmente em frascos plásticos devidamente identificados, contendo solução de formol a 10\%. Nos animais analisados, realizava-se uma marcação no pernil com lápis anilina para a posterior obtenção do peso da carcaça quente. Após a obtenção dos dados, estes eram digitados para o banco de dados para serem posteriormente analisados.

As amostras de pulmão foram encaminhadas ao Laboratório de Anatomia Patológica Professor Jefferson Andrade dos Santos, da Universidade Federal Fluminense, onde foram processadas de acordo com as técnicas habituais, incluídas em parafina e coradas pela Hematoxilina-Eosina. $\mathrm{Na}$ análise microscópica seguiram-se os critérios de classificação por escore descritos por Van Alstine et al., (1996), Irigoyen et al., (1998) e Scofano (2006). A reação peribronquial era graduada como escore zero quando não eram observados nódulos peribronquiais, de constituição mononuclear, indicando ausência de lesão; escore 1 quando pequenos nódulos estavam presentes em menos de $25 \%$ dos brônquios, bronquíolos e vasos; escore 2 quando pequenos nódulos estavam presentes em 25-75\% dessas estruturas e escore 3 quando estavam presentes em mais de $75 \%$ das estruturas ou devido à presença de grandes nódulos.

Foram considerados como negativos os pulmões com escores microscópicos 0 e 1 (Figuras $1 \mathrm{~A}$ e 1B) e positivos os que apresentaram escore microscópico 2 e 3 (Figuras. 1C e 1D).

Os resultados foram armazenados em banco de dados e analisados por meio do software Epilnfo 3.4 Windows, obtendo-se as médias de peso da carcaça quente dos animais dos grupos caso e controle, além da freqüência de casos positivos e negativos, tanto na macroscopia quanto na microscopia. A diferença entre as médias de peso foi calculada pelo Teste $t$ de Student para verificar se eram significantes. Obteve-se ainda o grau de significância por meio de Teste de McNemar com alfa igual a 5\% e a concordância pelo Índice de Kappa (ê) entre os exames macroscópico e microscópico (Thrusfield, 2004). 

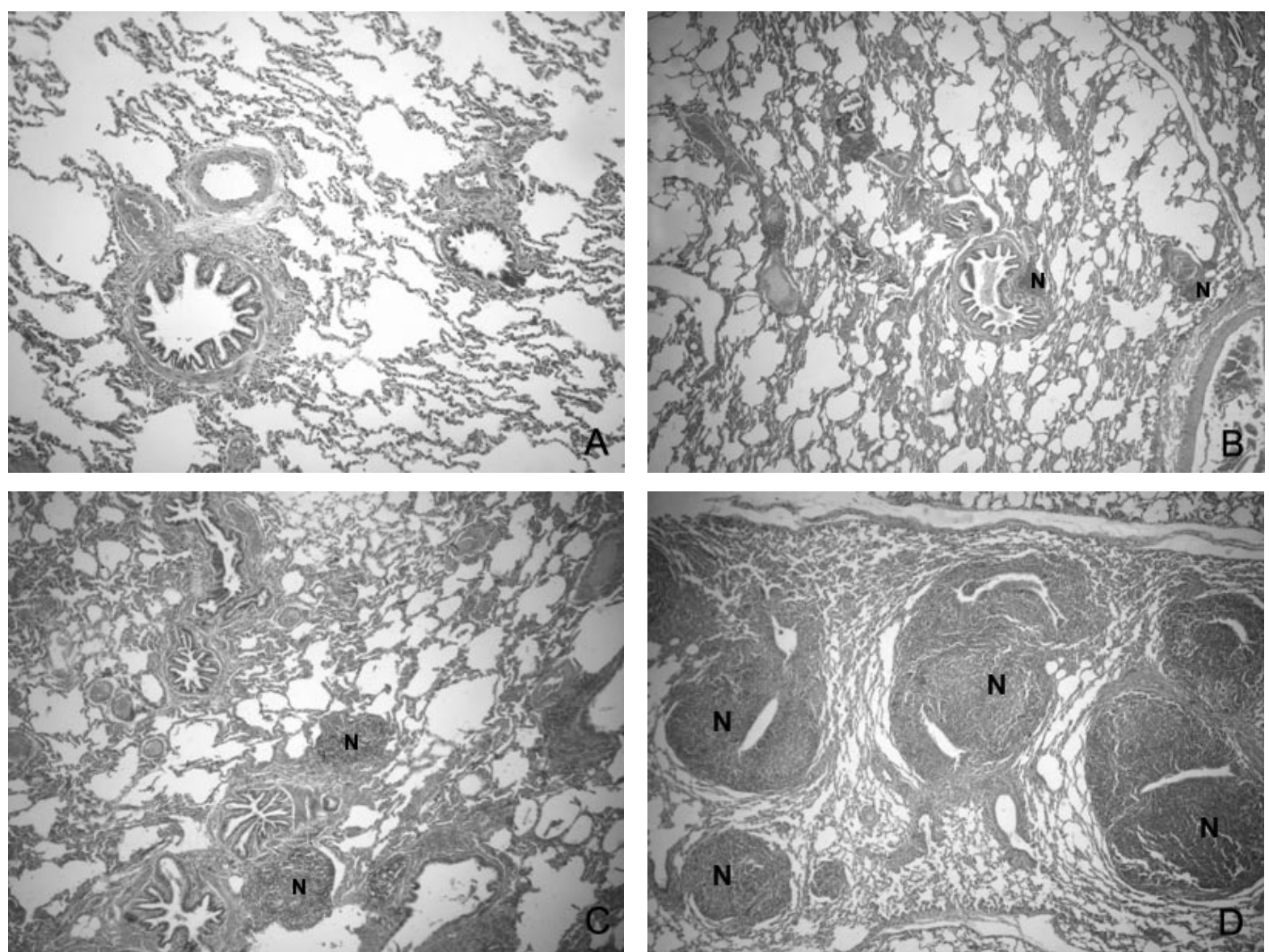

Figura 1: A) Suíno. Pulmão. Pneumonia Enzoótica Suína. Escore 0, ausência de lesões. H.E., Obj (4x). B) Suíno. Pulmão. Pneumonia Enzoótica Suína. Escore 1, pequenos nódulos de monocucleares $(\mathrm{N})$ em menos de $25 \%$ das estruturas. H.E., Obj (4x). C) Suíno. Pulmão. Pneumonia Enzoótica Suína. Escore 2, pequenos nódulos de mononucleares (N) em 25$75 \%$ das estruturas. H.E., Obj (4x). D) Suíno. Pulmão. Pneumonia Enzoótica Suína. Escore 3, presença de grandes nódulos (N). H.E., Obj (4x).

\section{Resultados e discussão}

A média de peso geral dos animais analisados foi de $84,33 \mathrm{~kg}$. Já as médias de peso de carcaça quente obtidas foram de $83,15 \mathrm{~kg}$ e $85,5 \mathrm{~kg}$ nos grupos caso (macroscopicamente positivos) e controle (macroscopicamente negativos) respectivamente. Observa-se, portanto, que animais do grupo controle pesaram $2,35 \mathrm{~kg}(85,50-83,15)$ a mais que os do grupo caso. No entanto, pela diferença das médias de peso entre os dois grupos pelo Teste T de Student, verificou-se que não houve diferença estatística $(P>0,05)$. Apesar de não significativa, essa redução é de $2,79 \%$ em relação ao peso médio de todos os animais analisados, sendo bastante expressiva economicamente, principalmente quando considerado o grande número de animais abatidos diariamente. Esse valor assemelha-se aos encontrados por Rautiainen e Wallgren (2001), que observaram variações de peso oscilando entre $2,8 \%$ e $44,1 \%$ em função da PES. Já Scofano (2006) observou uma diferença na média de peso entre animais macroscopicamente negativos e positivos de $9,57 \mathrm{~kg}$, equivalente a $11,90 \%(P<0,05)$ de perda em relação à média de peso de todos os suínos analisados, diferença bem maior do que a encontrada no presente estudo. No entanto, deve-se salientar que as observações realizadas ao abate por este autor ocorreram no final do inverno, sendo esta a estação do ano em que os animais estavam adquirindo peso, enquanto no presente estudo, as observações foram realizadas no início do outono, ou seja, os animais adquiriram peso principalmente no verão, época não favorável ao desenvolvimento de sinais clínicos que afetam ganho de peso. Visto que a PES é uma enfermidade que sofre influência sazonal (Dalla Costa et al., 2000; Silva, 1999) e as estações frias e secas do ano predispõem os animais a apresentarem sinais clínicos, que comprometem o ganho de peso (Stark et al. 1992), pode ser esta uma das razões que justifiquem essa pequena diferença de peso encontrada nos dois grupos.

Tabela 1: Relação entre os diagnósticos macroscópico e microscópico da PES em suínos abatidos sob Inspeção Federal no Oeste de Santa Catarina durante o mês de março de 2007, em um estudo tipo casocontrole*

\begin{tabular}{lccc}
\hline Casos & \multicolumn{2}{c}{ Casos Microscópicos } & \\
Macroscópicos & Negativos (\%) & Positivos (\%) & Total (\%) \\
\hline Negativos & $59(85,50)$ & $10(14,49)$ & $69(100)$ \\
Positivos & $17(24,63)$ & $52(75,36)$ & $69(100)$ \\
\hline Total & $76(55,07)$ & $62(44,92)$ & $138(100)$ \\
\hline
\end{tabular}

${ }^{*}$ Kappa $=62 \%$; McNemar não significativo $(P=0,248)$ 
Na Tabela 1 pôde-se comparar o grau de acerto no diagnóstico macroscópico do Fiscal Sanitário em relação ao diagnóstico microscópico. Verifica-se que dos animais diagnosticados como positivos para a PES na Inspeção Post-Mortem (macroscopia), 52 (75,36\%) apresentaram resultado positivo também na microscopia. A Inspeção Post-mortem (macroscopia) versus microscopia concordaram em 75,36\% dos diagnósticos positivos (52/69) e $85,5 \%$ dos negativos $(59 / 69)$, sendo $14,49 \%(10 / 69)$ e $24,63 \%$ os resultados discordantes negativos e positivos, respectivamente. Estas proporções quando analisadas mediante o Teste de McNemar, não indicaram diferenças significativas $(P>0,05)$, indicando haver similaridade entre ambos os tipos de diagnóstico. A taxa geral de concordância entre os dois métodos de diagnóstico foi de 80,44\%, e o valor de Kappa foi de 0,62\%, indicando que o nível de concordância entre os dois testes foi de $62 \%$, considerado "bom".

Apesar de não ser significante, há diferença entre os tipos de diagnóstico. Houve mais positivos pela macro do que pela micro, indicando o primeiro ser mais sensível e houve mais negativos na micro, indicando que esta é mais específica. A existência de resultados discordantes negativos (ausência de lesões macroscópicas, porém com alterações microscópicas) segundo Blanchard et al. (1996) ocorrem em casos em que o animal se infectou poucas semanas antes do abate e ainda não houve tempo para que as lesões se manifestassem na superfície do órgão. Outra possibilidade, segundo Van Alstine et al. (1996) e Irigoyen et al. (1998), referese aos animais que se infectaram, porém tiveram regressão espontânea das lesões superficiais. Segundo Kobisch et al. (1993), a recuperação das lesões pode ocorrer por volta de 812 semanas após a exposição. Desta forma, justifica-se a ausência das lesões macroscópicas na superfície do órgão, porém, com alterações microscópicas.

Em contrapartida, a existência de resultados discordantes positivos (pulmões com lesões sugestivas de PES na macroscopia e ausência de lesões características na microscopia) indica dificuldades em reconhecer as lesões características da enfermidade em função de outras lesões pulmonares presentes na superfície do órgão, como por exemplo, em função de um quadro de atelectasia, que também causa áreas de depressão e escurecimento do parênquima pulmonar (Jones et al., 2000), parecido com as lesões

\section{Referências}

ARMSTRONG, C.H. Porcine Mycoplasmas. In: WHITFORD, H.W.; ROSENBUSH, R.F.; LAUERMAN, L.H. (Eds.). Mycoplasmosis in animals: laboratory diagnosis. Ames: lowa State University, 1994.

BAHNSON, P.B.; MARSH, W.E.; DIAL, G.D. The associations among serology to $M$. hyopneumoniae, lung lesions at slaughter and a clinical index of cough in groups of growing pigs. INTERNATIONAL CONGRESS ON PIG VETERINARY SOCIETY, 1994, Bangkok. Proceedings..., Bangkok. 1994. p. 131. (Resumo).

BLANCHARD, B.; KOBISCH, M.; BOVÉ, J.M. et al. Polymerase chain reaction for Mycoplasma hyopneumoniae detection in tracheobronchiolar washings from pigs. Mol. Cell. Prob., v. 10, p. 15-22, 1996.

DALLA COSTA, O.A.; MORES, N.; SOBESTIANSKY, J. et al. Fatores de risco associados à rinite atrófica progressiva e pneumonias crônicas nas fases de crescimento e terminação. Concórdia: EMBRAPA-CNPSA, 2000. 4 p. (EMBRAPA-CNSA. Comunicado técnico, 267). macroscópicas causadas pela PES. Além disso, alterações do aspecto macroscópico podem ocorrer em virtude de operações tecnológicas do abate mal realizadas, como por exemplo, aspiração de sangue durante a sangria, que irá causar congestão do órgão, prejudicando, dessa maneira, que as lesões da enfermidade sejam corretamente identificadas na inspeção sanitária.

Como o número de diagnósticos positivos discordantes foi maior do que o número de discordantes negativos, ou seja, casos que foram considerados positivos pela Inspeção Sanitária, eram na verdade negativos à histopatologia, pode também ter contribuído para que a diferença estatística de peso entre os dois grupos não fosse significativa, influenciando o número real de animais com PES, que tem peso menor. Além desta possibilidade e da influência da estação do ano no ganho de peso dos animais, outra hipótese que deve ser apontada é o tamanho da amostra, que se fosse maior, poderia reduzir a margem de erro.

Em função do número de diagnósticos positivos discordantes $(24,63 \%)$ ter sido maior que o número de negativos, recomenda-se que os fiscais sanitários que realizam a detecção das lesões nas linhas, se dediquem mais neste momento, para evitar que outros tipos de pneumonias e lesões pulmonares que se superpõem sejam confundidas com as da referida enfermidade. Além disso, deve-se atentar para o fato de que as manobras tecnológicas no abate sejam conduzidas corretamente, observando as normas previamente estipuladas pelos órgãos competentes. Desta maneira, minimiza-se o risco de erro nos dados obtidos e registrados nos mapas de condenação do Serviço de Inspeção Sanitária, visto que estes são amplamente utilizados para estabelecer estratégias eficientes de controle nos rebanhos, bem como em estudos epidemiológicos da doença em determinada região.

\section{Conclusão}

De acordo com os resultados, pode-se concluir que não há diferença significante no diagnóstico de PES quando se compara os diagnósticos da Inspeção Sanitária post-mortem (macroscopia) com o histopatológico (microscopia). E, finalmente, observou-se que a diferença de peso da carcaça quente entre animais do grupo controle $(85,5 \mathrm{Kg})$ foi maior que a do grupo caso $(83,15 \mathrm{Kg})$, embora não tenha sido estatisticamente significativa.

IRIGOYEN, L.F.; ALSTINE, W.V.; TUREK, J. et al. Ultrastructural observation of the airways of recovered and susceptible pigs after inoculation with Mycoplasma hyopneumoniae. Pesq. Vet. Bras., v. 18, n. 1, p. 1-7, 1998.

JONES, T. C.; HUNT, R. D.; KING, N. W. Patologia Veterinária. 6. ed. São Paulo: Manole, 2000. p. 970-976.

KOBISCH, M.; BLANCHARD, B.; LE POTIER, M.F. Mycoplasma hyopneumoniae infection in pigs duration of the disease and resistance. Vet. Res., v. 24, p. 67-77, 1993.

MAES, D.G.; DELUYKER, H.; VERDONCK, M. et al. Non-infectious factors associated with macroscopic and microscopic lung lesions in slaughter pigs from farrow-to-finish herds. Vet. Rec., v. 148, p. 41-46, 2001.

MARTÍNEZ, J.; JARO, P.J.; ADURIZ, G. et al. Carcass condemnation causes of growth retarded pigs at slaughter. Vet. J., v. 174, n. 1, p. 160-164, jul. 2007. 
MORRIS, C.R.; GARDNER, I.A.; HIETALA, S.K. et al. Enzootic pneumonia: comparison of cough and lung lesions as predictors of weight gain in swine. Prev. Vet. Med., v. 59, p. 197-204, 1995.

MOUSING, J.; LYBYE, H.; BARFOD, K. et al. Chronic pleuritis in pigs for slaughter: an epidemiological study of infectious and rearing systemrelated risk factors. Prev. Vet. Med., v. 9, p. 107-119, 1990.

PIFFER, I.A. Doenças respiratórias: aspectos clínicos e controle. In: SIMPÓSIO GOIANO DE SUINOCULTURA, 1997, 1., Goiânia. Anais..., Goiânia, 1997, p. 70. (Resumo).

RAUTIAINEN, E.; WALLGREN, P. Aspects of the Transmission of Protection against Mycoplasma hyopneumoniae from Sow to Offspring. J. Vet. Med. Série B, v. 48, p. 55-65, 2001.

SCOFANO, A.S. Pneumonia Enzoótica suína: diagnóstico anátomopatológico, prevalência e efeitos sobre o desenvolvimento da carcaça. 2006. 51 f. Dissertação (Mestrado) — Faculdade de Veterinária Universidade Federal Fluminense, Niterói, 2006.

SILVA, A.J. Doenças respiratórias. Rev. Suin. Ind., n. 137, fev.-mar., 1999.

SOBESTIANSKY, J.; BARCELLOS, D.; MORES, N. et al. (Ed.).Clínica e patologia suína. 2. ed. Goiânia: Art 3 Impressos Especiais, 1999, 464 p.
SOBESTIANSKY, J.; BARBARINO JÚNIOR, P.; HIROSE, F. et al. Pneumonia enzoótica suína: prevalência, impacto econômico, fatores de risco e estratégias de controle. Goiânia: Art 3 Impressos Especiais, 2001, 44 p.

STARK, K.D.C.; KELLER, H.; EGGENBERGER, E. Climatic conditions and airborne transmission of $M$. hyopneumoniae. In: IPVS CONGRESS, $1992,12^{\text {th }}$. Proceedings...1992, p. 559.

THRUSFIELD, M. Epidemiologia veterinária. 2. ed. São Paulo: Roca, 2004, p. 596.

VAN ALSTINE, W.G.; STEVENSON, G.W.; KANITZ, C.L. Porcine reproductive and respiratory syndrome virus does not exacerbate Mycoplasma hyopneumoniae infection in young pigs. Vet. Microbiol., v. 49, p. 297-303, 1996.

WALKER, P.K.; BILKEI, G. Tail-biting in outdoor pig production. Vet. J., v. 171, p. 367-369, 2006.

ZIMMERMANN, W.; PLONAIT, H.; Erkrankungen des Atmungsapparates. In: PLONAIT, H.; BICKHARDT, K. (Ed.). Lehbuch der Schweinekrankheiten. Berlin:Parey Buchverlag, 1997, p.111-150. 